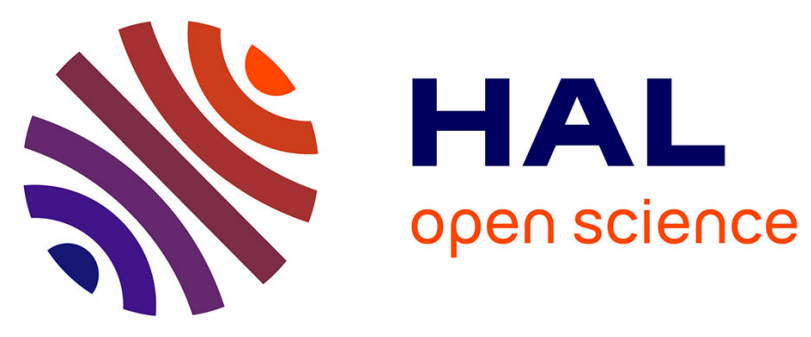

\title{
Flexible Call Admission Control with Preemption in LTE Networks
}

\author{
Aymen Belghith, Nesrine Turki, Bernard Cousin, Mohammad S. Obaidat
}

\section{To cite this version:}

Aymen Belghith, Nesrine Turki, Bernard Cousin, Mohammad S. Obaidat. Flexible Call Admission Control with Preemption in LTE Networks. IEEE International Conference on Communications (ICC'16), Mobile and Wireless Networking Symposium, May 2016, Kuala Lumpur, Malaysia. 10.1109/ICC.2016.7511019 . hal-01285168

\section{HAL Id: hal-01285168 \\ https://hal.science/hal-01285168}

Submitted on 8 Mar 2016

HAL is a multi-disciplinary open access archive for the deposit and dissemination of scientific research documents, whether they are published or not. The documents may come from teaching and research institutions in France or abroad, or from public or private research centers.
L'archive ouverte pluridisciplinaire HAL, est destinée au dépôt et à la diffusion de documents scientifiques de niveau recherche, publiés ou non, émanant des établissements d'enseignement et de recherche français ou étrangers, des laboratoires publics ou privés. 


\title{
Flexible Call Admission Control with Preemption in LTE Networks
}

\author{
Aymen Belghith ${ }^{1}$, Nesrine Turki ${ }^{1}$, Bernard Cousin ${ }^{2}$, and Mohammad S. Obaidat, ${ }^{3}$ Fellow of IEEE and Fellow of SCS \\ ${ }^{1}$ University of Sfax, Road of Aeroport Km 0.5, 3029 Sfax, Tunisia, Email: aymen.belghith@gmail.com \\ ${ }^{2}$ University of Rennes I - IRISA, Campus de Beaulieu, 35042 Rennes Cedex, France, Email: bernard.cousin@univ-rennes1.fr \\ ${ }^{3}$ Fordham University, 441 East Fordham Road, JMH 340, Bronx, New York 10458, Email: mobaidat@fordham.edu
}

\begin{abstract}
This paper introduces a new call admission control (CAC) mechanism for Long Term Evolution (LTE) networks supporting multimedia services with different classes of traffic. Our CAC mechanism classifies calls into real time and non-real time users, then estimates the channel quality based upon the received signal strength (RSS) value, and finally identifies the call as either new call $(\mathrm{NC})$ or handoff call $(\mathrm{HC})$ request before performing admission control decision. We also use a simple preemption technique in order to allocate the resources to high priority bearer requests. We show through extensive simulation analysis that our CAC mechanism provides high number of accepted users with higher priorities while providing high system throughput.
\end{abstract}

Keywords-LTE; CAC; Channel state; QoS; Preemption; New call; Handoff call.

\section{INTRODUCTION}

The growing demand for network services, such as voice over Internet Protocol (VoIP), web browsing, video telephony and video streaming with time constraints and bandwidth (BW) requirements poses new challenges in the design of cellular networks for future generations. The Third Generation Partnership Project (3GPP) introduced Long Term Evolution (LTE) as a response to this need, with ambitious performance targets and defined an all-IP radio access. The Radio Resource Control (RRC) layer is the most important layer in the signaling process. This layer supports several key features between the user equipment (UE) and the evolved NodeB (eNodeB) such as the connection management [1].

Orthogonal Frequency Division Multiple Access (OFDMA) and Single-Carrier Frequency Division Multiple Access (SCFDMA) are the access technologies used for the downlink and uplink directions. The LTE systems use radio frequency bands with a width varying from $1.4 \mathrm{MHz}$ to $20 \mathrm{MHz}$. For example, when using a $20 \mathrm{MHz}$ band, a theoretical data rate of up to $300 \mathrm{Mbit} / \mathrm{s}$ in the downlink direction can be performed. The fourth generation (4G), called LTE Advanced (LTE-A) can offer a download speed of up to $1 \mathrm{Gbit} / \mathrm{s}$ when using frequency bands of $2 \times 100 \mathrm{MHz}$ wide which are defined in 3GPP Releases 10 [2] and 11 [3].

There are already more than 240 million LTE mobile phone users in the world (2014 data) and it is expected that this number reaches 2.6 billion of LTE users by the end of 2019
[4]. For this reason, both communities of research and industry have done a considerable effort on the study of LTE systems, offering new and innovative solutions to analyze and improve their performance in order to effectively deal with the huge number of mobile user calls. Moreover, efficient mechanisms have to also consider the diversity of services which produce a diversity in the Quality of Service (QoS) requirements.

For this reason, admission control mechanisms are used by operators to ensure continuous service quality supply and to provide high levels of satisfaction for customers while using the network bandwidth optimally. An admission control mechanism decides if it accepts or rejects a call taking into account one or many parameters such as network conditions, resources use, and types of services.

In this paper, we propose an admission control mechanism where users are classified into different traffic classes. These users have different priorities indicated by the Channel Quality Indicator (QCI), which varies according to the class of traffic. Admission control for these LTE users depends on several parameters such as traffic classes, available radio resources, channel quality, and call type. We then compare our proposal with other CAC mechanisms proposed for the LTE network.

This work is organized as follows. In Section II, we describe admission control mechanisms already proposed for LTE as well as our proposal. In Section III, we present our LTE system model. We evaluate the different admission control mechanisms in Section IV through extensive simulations. Finally we conclude our work in Section V.

\section{CALL ADMISSION CONTROL MECHANISMS}

To simultaneously meet the bandwidth and QoS requirements, admission control mechanisms are used by network operators as a method for continuous supply of quality of service. The lack of an adequate admission control is partly responsible for the current difficulties in the telecommunications industry. In this section, we provide a state of the art of existing admission control mechanisms in LTE networks before presenting our own mechanism called Flexible Call Admission Control (FCAC). Note that a preemption algorithm can be added to FCAC and the resulting mechanism is called FCAC with preemption (FCAC_P). 


\section{A. Basic Call Admission Control (BCAC)}

Basic Call Admission Control (BCAC) is a static admission control mechanism [5]. The decision of the acceptance or rejection of the call is based only on the availability of radio resources. Its principle is to determine if there is sufficient bandwidth (BW) to accept this call. The unit of allocation in LTE networks is the Physical Resource Block (PRB). Therefore, to adapt BCAC to LTE networks, we propose that the eNodeB calculates the number of PRBs required for the call. Then, it compares this number with the number of available PRBs. The call is accepted only if there are enough available radio resources.

\section{B. Multi-Service Call Admission Control (MSCAC)}

Multi-Service Call Admission Control (MSCAC) was proposed for 3G/4G networks [6]. Two types of service classes are defined: Real Time (RT) for conversational and streaming calls and Non-Real Time (NRT) for Best Effort calls (see Figure 1). MSCAC divides the resources into two parts: a part for NRT calls and a second part for RT calls. An NRT call is accepted only if there are enough available PRBs in the BW part for NRT.

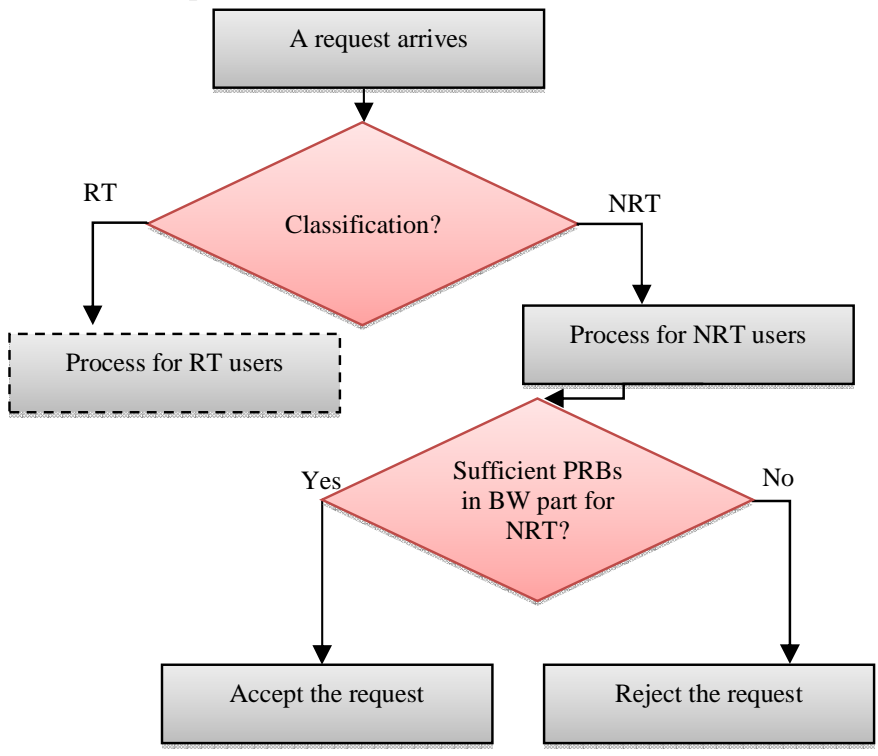

Fig. 1. General scheme of MSCAC

To provide efficiency in LTE networks, operators can favor RT calls by increasing BW part for RT. Moreover, MSCAC takes into account the quality of the channel during CAC decision and classifies RT calls into Voice over Internet Protocol (VoIP) or Video calls. The PRBs reserved for RT calls are divided into three parts:

- A BW part for RT calls having bad channel.

- A BW part for RT calls having good channel and classified into Video calls.

- A BW part for RT calls having good channel and classified into VoIP calls.

An RT call having bad channel estimation is accepted only if there is enough free bandwidth in BW part for bad channel (see Figure 2). The channel quality is considered as bad when the channel quality estimation is below a defined received signal strength threshold $\left(\mathrm{RSS}_{\mathrm{th}}\right)$. If the channel quality estimation is good, the RT call is categorized into either New Call (NC) or Handoff Call (HC). RT HCs have higher priority than RT NCs and therefore they are treated first. Note that the process is the same for $\mathrm{HC}$ and NC.

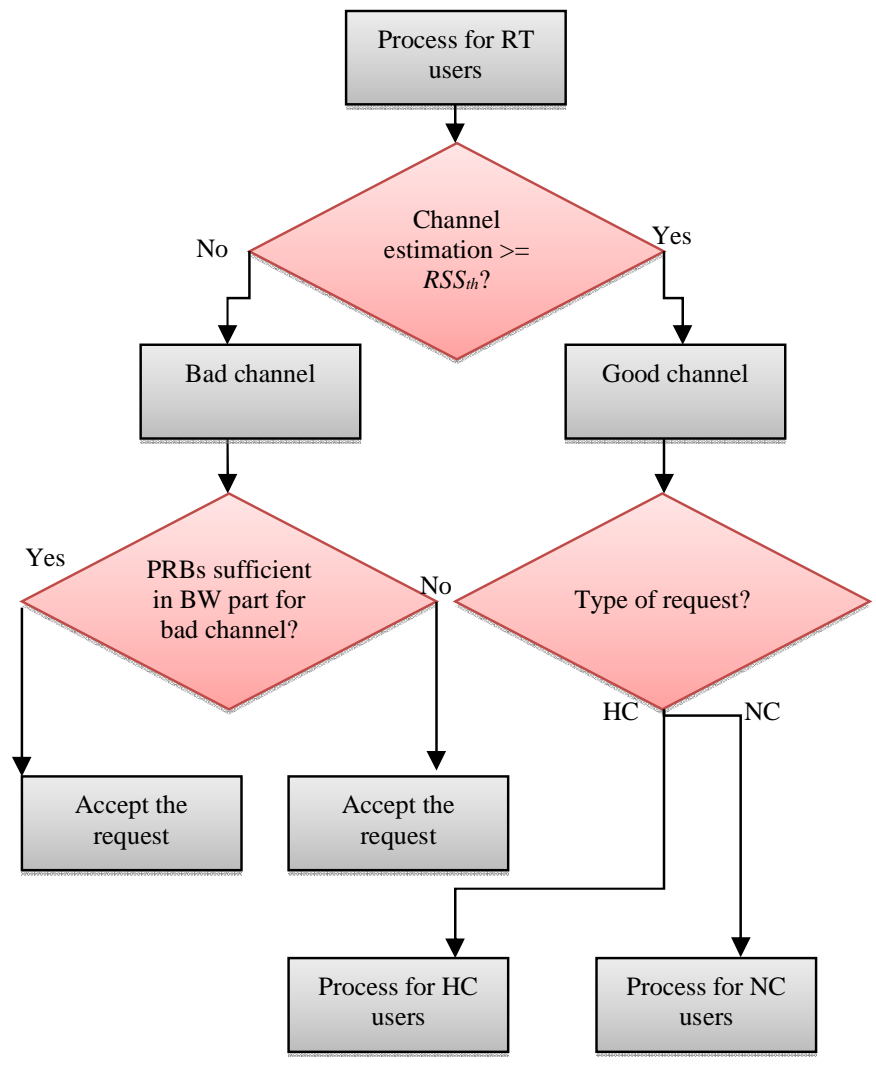

Fig. 2. Process for RT users in MSCAC

Finally, the RT call is classified into either VoIP call (having the highest priority) or Video call. When there are sufficient free PRBs in the BW part for VoIP, the VoIP call is accepted. Otherwise, the eNodeB checks if there are enough free PRBs in the BW part for bad channel. Therefore, a VoIP call is rejected only if there are not enough PRBs in BW parts for VoIP and bad channel (see Figure 3).

In the same way, a Video call is rejected only if there are not enough PRBs in BW parts for Video and bad channel. Note that Video calls having the smallest tolerance are accepted first.

\section{Channel Based Efficient Call Admission Control (CBECAC)}

An admission control mechanism, called Channel Based Efficient CAC (CBECAC), is presented in [7]. A call is classified into either RT or NRT call. The RT call is classified into either $\mathrm{HC}$ or NC. The PRB is divided into three parts: 
- A part for NRT calls.

- A part for RT new calls.

- A part for RT handoffs calls.

An NRT call is accepted only if there are enough free PRBs in the BW part for NRT. After classifying the RT call, an RT $\mathrm{NC}$ is accepted when there are enough free PRBs in the BW part for NC. Otherwise, a preemption algorithm is performed on NRT calls.

An RT HC is accepted if the reserved bandwidth for RT HC is available. Therefore, the NRT calls are preempted only for RT NC calls.

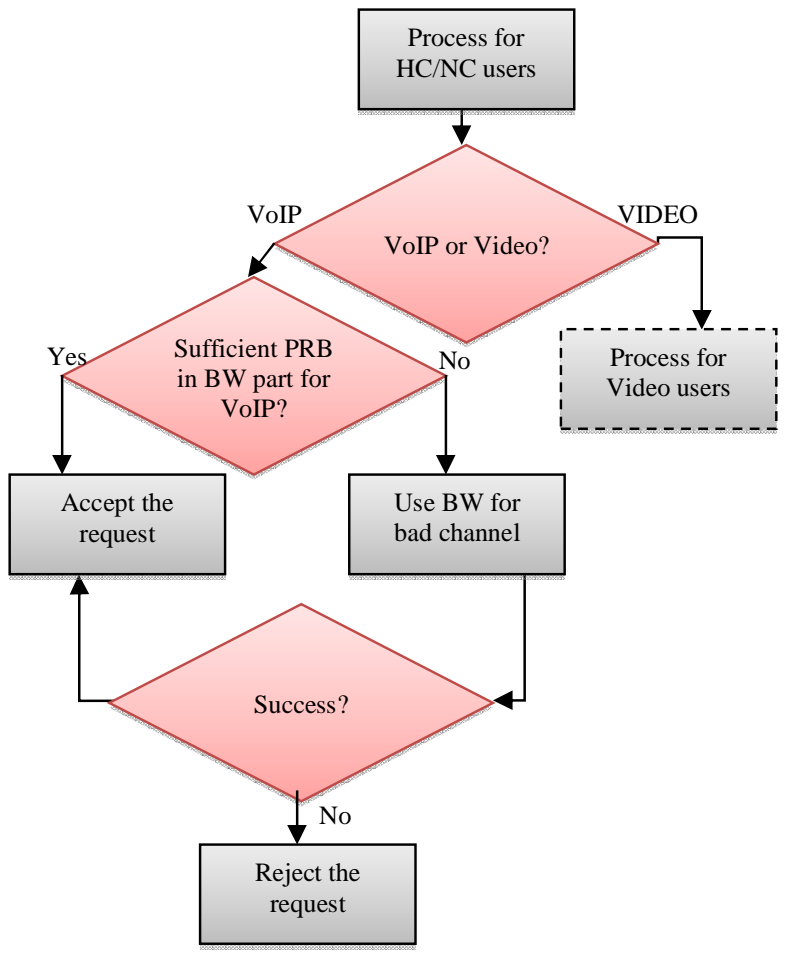

Fig. 3. Process of RT HC/NC when the channel quality is good in MSCAC

\section{Synthesis of existing CAC algorithms}

Note that BCAC is a classical CAC that processes all calls in the same way and therefore it cannot consider the type of call and the channel quality. Therefore, BCAC is not efficient in LTE networks as the throughput cannot be high and QoS requirements cannot be met.

MSCAC considers the channel quality as well the type of call in its CAC decision. Moreover, it favors HCs. However, when there are not sufficient free PRBs in the BW part for $\mathrm{HC}$, the eNodeB checks the BW part for bad channel quality instead of the BW part for NRT. Therefore, the eNodeB favors NRT calls on RT calls having bad channel quality.

Finally, CBECAC considers the type of the call. However, the channel quality is not taken into account and so this algorithm cannot provide high throughput. Moreover, with this CBECAC algorithm, the eNodeB favors NCs on HCs. In the next section, we present our proposal that aims to overcome the drawbacks of existing CAC algorithms.

\section{E. Proposed CAC Mechanim}

In this paper, we propose a flexible Call Admission Control scheme with preemption mechanism that takes into account the channel quality, the LTE allocation unit (PRB), and the QoS classes. First, we present our flexible CAC (FCAC). Then we describe the preemption algorithm applied to FCAC. Recall that when performing the preemption algorithm, the mechanism is called FCAC_P.

\section{1) $F C A C$}

FCAC works as follows. First, a call is classified into either NRT or RT call. First of all, we consider NRT calls. If the total number of available PRBs is insufficient to fulfill the number of PRBs requested by the NRT call, the request is rejected. Otherwise, if the occupation ratio of the bandwidth $\left(O R \_B W\right)$ is lower than a defined threshold for NRT calls, called th_NRT, the NRT call is immediately accepted. The occupation ratio of $\mathrm{BW}$ represents the ratio between the number of PRBs already reserved and the total number of PRBs. Otherwise, the NRT call is blocked with a probability, called blocking rate for NRT calls $\left(B R \_n r t\right)$, see Figure 4.

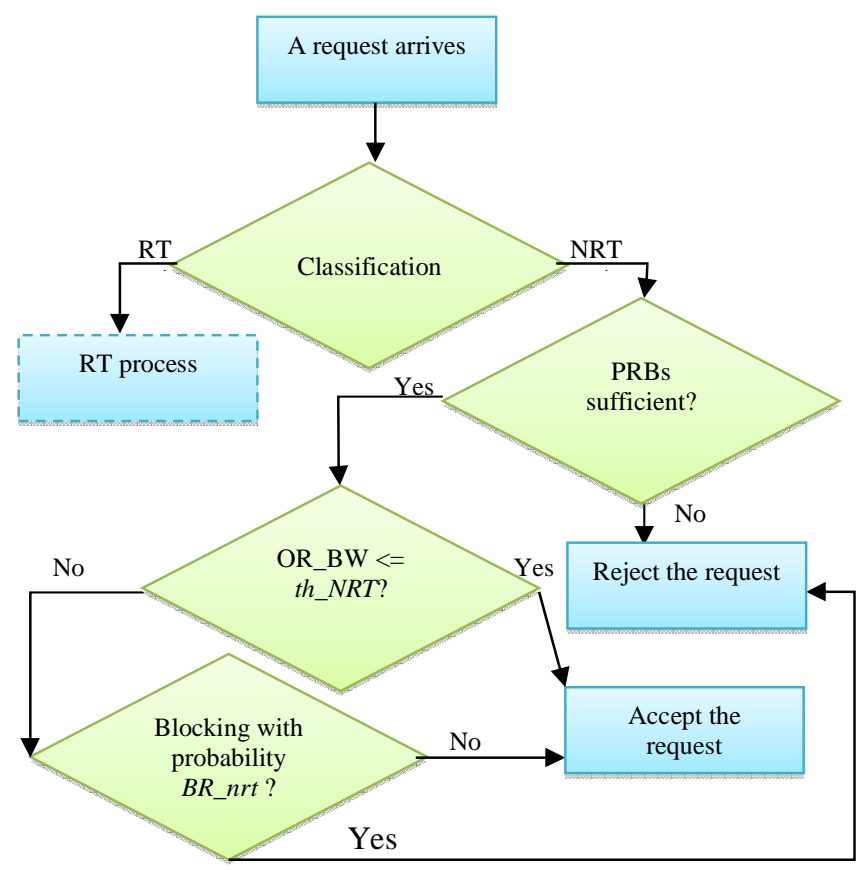

Fig. 4. General scheme in FCAC

The aim of using BR_nrt is to give the possibility to accept an NRT call when there are available PRBs even if the threshold of BW reserved to NRT calls is reached. Moreover, this parameter provides flexibility to the operator by choosing the suitable value. For example, a high value of $B R \_n r t$ favors RT users. 
Now, we consider RT calls. First, we compare the quality of the channel with a defined RSS threshold ( $\left.\mathrm{RSS}_{\mathrm{th}}\right)$. If the RT user has a bad channel, we propose that the RT call is accepted only if $O C_{-} B W$ is lower than a threshold, called $t h \_R T \_B C$ (see Figure 5).

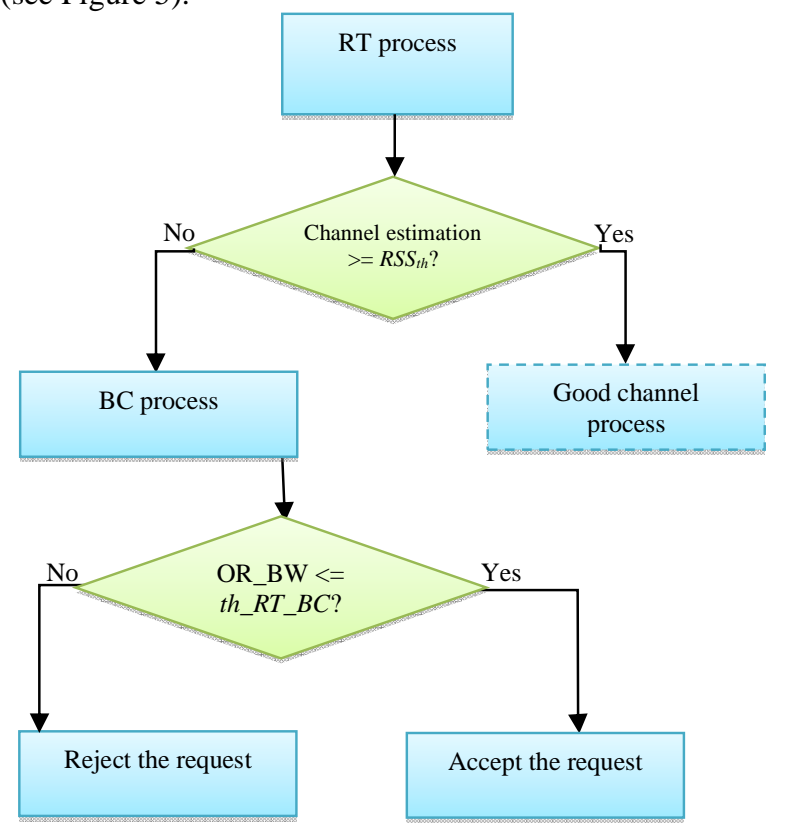

Fig. 5. RT process in FCAC

If the channel quality is good then we check if we have enough available PRBs to accept this request. If resources are available then we check if a threshold, called $t h \_R T \_N C$, has been exceeded. If $t h \_R T \_N C$ is exceeded then we identify whether the request is RT HC or RT NC. If the RT call is considered as $\mathrm{HC}$ then it is automatically accepted because $\mathrm{HC}$ has the highest priority.

If the RT call is considered as NC, it is accepted but with a blocking rate probability called $B R n c \_r t$. The higher $B R n c \_r t$ the more handoff calls are favored. If the threshold for the use of the bandwidth is not exceeded, then all RT calls are treated in the same way and accepted (see Figure 6).

2) $F C A C_{-} P$

FCAC_P adds to FCAC a preemption algorithm in order to favor RT calls. Our proposed preemption algorithm works as follows. The eNodeB browses accepted NRT calls and not already preempted. We propose to do not preempt a defined number of NRT calls, called Number of non-preempted NRT users (Nnp_NRT), in order to do not totally reject NRT calls.

We also choose to do not preempt recent calls as the old calls have partially served. If the number of PRBs acquired after preemption is sufficient to accept RT calls, then preemption is executed. Otherwise, it will be canceled.

\section{SYSTEM MODEL}

\section{A. Radio Resources}

The LTE downlink frame duration is $10 \mathrm{~ms}$. Each downlink frame contains 10 subframes of $1 \mathrm{~ms}$ duration.

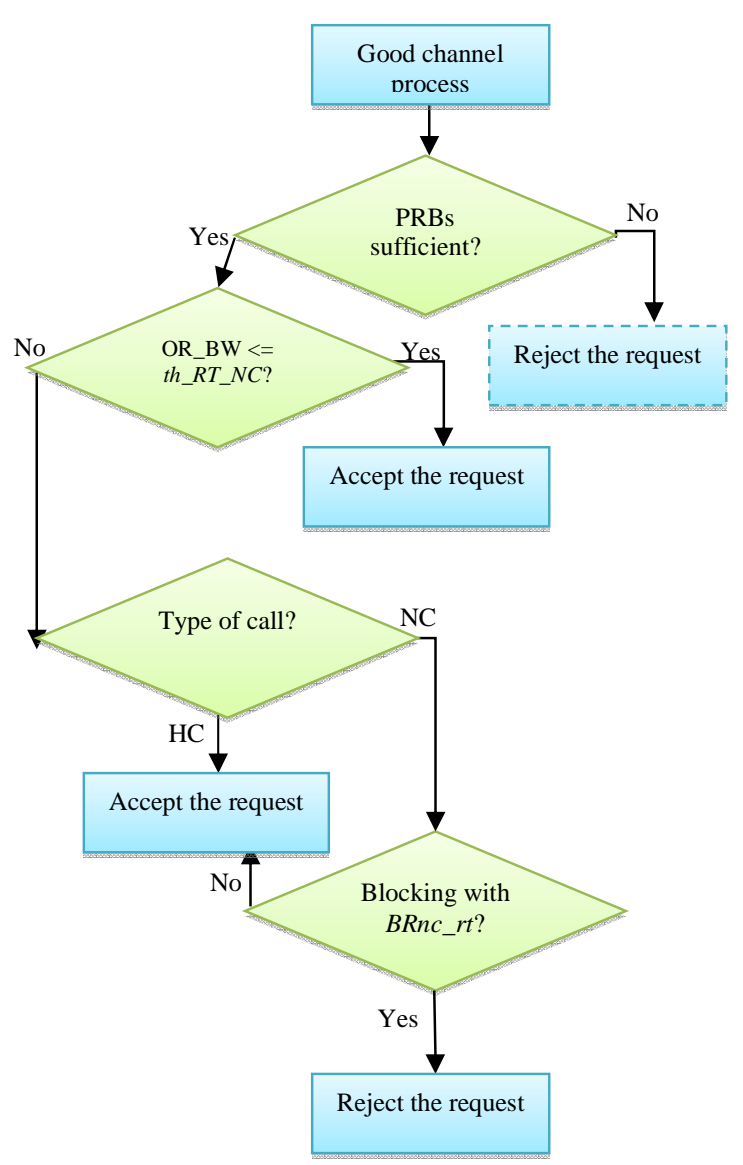

Fig. 6. Process of RT users having good channel in FCAC Each subframe consists of two slots. In the frequency domain, the resources are grouped into 12 subcarriers. They occupy a total of $180 \mathrm{KHz}$ with a spacing of $15 \mathrm{KHz}$. A unit of 12 subcarriers in a period of one slot is called a physical resource block (PRB). A PRB contains 84 (resp. 72) Resource Elements (REs) in the case of a normal (resp. short) cyclic prefix (see Figure 7).

\section{B. User SINR and Modulation and Coding Scheme}

The evaluation of the channel condition is based on the estimation of the signal (Signal-to-Interference plus Noise Ratio: SINR) of the UE. For each PRB, the effective SINR (SINReff) is used as a metric to evaluate the channel quality indications. The SINR value of subcarrier $n$ is calculated as follows:

$$
\operatorname{SINR}_{n}=\mathrm{P}_{\mathrm{n}} \mathrm{s} /\left(\mathrm{N}_{0} \times \mathrm{W}_{\mathrm{sc}}+\Sigma_{\mathrm{i} \neq \mathrm{s}} \mathrm{P}_{\mathrm{n}}^{\mathrm{i}}\right)
$$

Where $n$ represents the index of the sub-carrier, $P n^{s}$ represents the received power of the serving eNodeB for subcarrier $n, N_{0}$ represents the noise density, and $W_{s c}$ represents the frequency spacing.

Effective SINR is calculated using the average instantaneous capacity (MIC). This method is described in the following equation [8]:

$$
\mathrm{SINR}_{\mathrm{eff}}=2^{\mathrm{MIC}}-1
$$




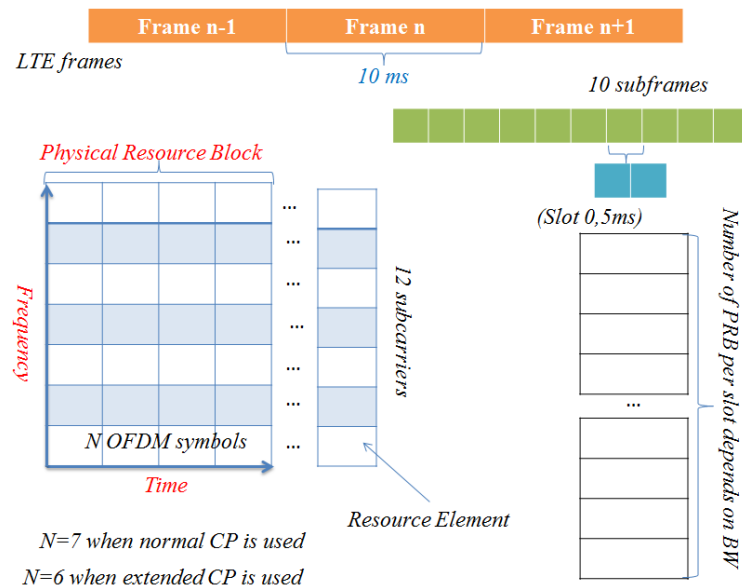

Fig. 7. Radio resources structure

Where the MIC is calculated by averaging the capacity of all the $N$ subcarriers of a PRB:

$$
M I C=1 / N \sum^{N}{ }_{n=1}^{\prime} \log _{2}\left(1+\operatorname{SINR}_{n}\right)[\text { in bps } / \mathrm{Hz}]
$$

The association between the Modulation and coding Scheme (MCS) and effective SINR is performed according to Table I [9].

\section{User classification}

We characterize the users into two categories; Real-Time (RT) and Non-Real Time (NRT). We consider QCIs equals to 1, 4, 8 or 9 . Table II shows the different types of services suggested for each level with the corresponding QCI values as well as some examples of services used [10].

The RT type represents the most privileged users and uses the Guaranteed Bit Rate (GBR) type. GBR means that a minimum of bit rate resources has to be reserved. NRT users have limited access to services and uses Non-Guaranteed Bit Rate (Non-GBR) [11].

\section{SIMULATION RESULTS}

In this section, we present the simulation results in order to evaluate the performance of our proposed CAC mechanisms (FCAC and FCAC_P). They are compared with three existing CAC mechanisms: BCAC, MSCAC, and CBECAC. First, we present our simulation model. Then we present the simulation results showing how optimal results are obtained from FCAC_P.

The same optimization approach is followed for other existing CAC mechanisms in order to maximize the number of RT calls accepted while trying to maximize the throughput of system. Finally, we make a comparison between different LTE CAC mechanisms.

\section{A. Simulation Model}

We consider a simulation model based on one hexagonal cell having a radius of $1500 \mathrm{~m}$. The bandwidth used is 20 $\mathrm{MHz}$ and therefore there are $100 \mathrm{PRB}$ per slot.
TABLE I. CHANNEL QUALITY AND NUMBER OF BITS

TRANSMITTED PER PRB FOR VARIOUS MCS.

\begin{tabular}{|l|l|l|}
\hline MCS & $\begin{array}{l}\text { SINR } \\
\text { interval }(\mathrm{dB})\end{array}$ & $\begin{array}{l}\text { Number of bits } \\
\text { transmitted per PRB }\end{array}$ \\
\hline QPSK 1/2 & {$[2.9,6.3[$} & $7 * 12 * 2 * 1 / 2=84$ \\
\hline QPSK 3/4 & {$[6.3,8.6[$} & $7 * 12 * 2 * 3 / 4=126$ \\
\hline 16QAM $1 / 2$ & {$[8.6,12.7[$} & $7 * 12 * 4 * 1 / 2=168$ \\
\hline 64QAM $3 / 4$ & {$[12.7,16.9[$} & $7 * 12 * 4 * 3 / 4=252$ \\
\hline 64QAM 2/3 & {$[16.9,18[$} & $7 * 12 * 6 * 2 / 3=336$ \\
\hline 64QAM $3 / 4$ & {$[18, \infty[$} & $7 * 12 * 6 * 3 / 4=378$ \\
\hline
\end{tabular}

TABLE II. CHARACTERISTICS OF USERS CLASS.

\begin{tabular}{|c|c|l|}
\hline User class & Authorized QCI & Examples of services \\
\hline RT & 1 and 4 & $\begin{array}{l}\text { Real-time gaming and } \\
\text { conversational voice, } \\
\text { respectively }\end{array}$ \\
\hline NRT & 8 and 9 & $\begin{array}{l}\text { IP Multimedia } \\
\text { Subsystem (IMS) } \\
\text { signaling, respectively }\end{array}$ \\
\hline
\end{tabular}

TABLE III. OPTIMAL PARAMETERS VALUES FOR CAC MECHANISMS

\begin{tabular}{|l|l|}
\hline CAC mechanisms & Optimal parameters values \\
\hline BCAC & \multicolumn{1}{|c|}{---} \\
\hline MSCAC & BW part for NRT $=40 \%$ \\
& BW part for bad channel $=20 \%$ \\
& BW part for VoIP $=20 \%$ \\
& BW part for Video $=20 \%$ \\
\hline CBECAC & BW part for NRT $=40 \%$ \\
& BW part for RT NC/HC $=30 \%$ \\
& Nnp_NRT $=2$ \\
\hline FCAC & th_NRT $=50 \%, t h \_R T \_B C=$ \\
& $60 \%, t h \_R T \_N C=90 \%, R S S_{\text {th }}=$ \\
& $8.6 \quad$ dB, BR_nrt = $=.2, \quad$ and \\
& $B R n c \_r t=0.01$ \\
\hline FCAC_P & In addition to the parameters of \\
& FCAC,Nnp_NRT $=2$ \\
\hline
\end{tabular}

The number of sub-carriers per PRB is 12 . The classes of service are distributed respectively between RT and NRT with probability $2 / 3$ and $1 / 3$. A QCI is chosen randomly between the two allowed values (see Table II). The rate requested for each user depends on the service type. The detailed simulation parameters were presented in [12].

\section{B. Simulation Parameters}

In this section, we present the simulation results when varying $B R \_n r t, B R n c \_r t$, and $N n p \_n r t$. Note that we have investigated all parameters of our proposed CAC mechanisms as well as those of BCAC, MSCAC, and CBECAC. All optimal values are presented in Table III.

\section{Investigation of FCAC_P Parameters}

\section{1) Investigation of $B R \_N R T$}

Figure 8 represents the accepted NRT and RT calls as a function of $B R \_n r t$. First, we consider the NRT calls. Note that $B R \_n r t$ is the blocking rate for NRT users. 


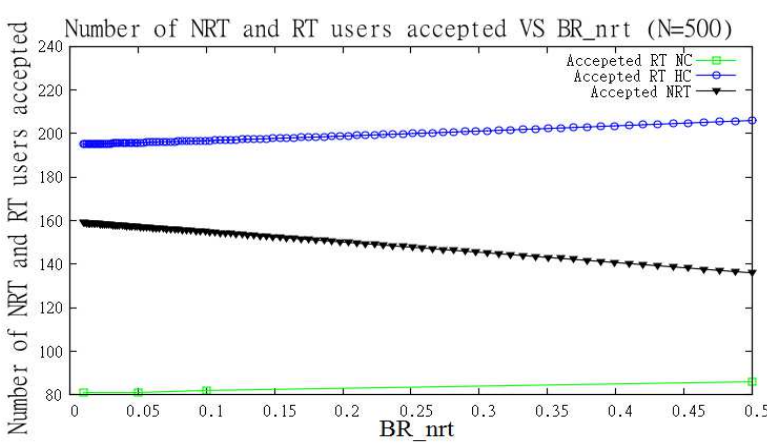

Fig. 8. Number of NRT and RT users accepted Vs $B R \_n r t$

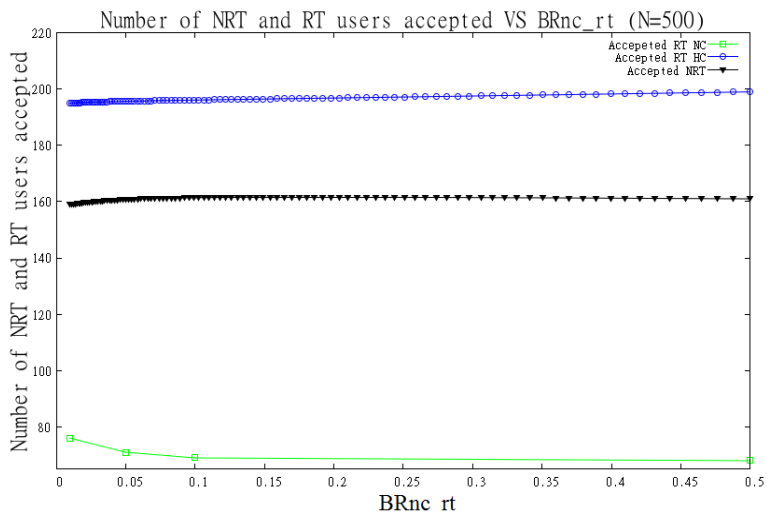

Fig. 9. Number of NRT and RT users accepted Vs $B R \_n c \_r t$

We verify that the number of NRT calls accepted decreases when $B R \_n r t$ increases. We note that for an increase of $B R \_n r t$ from 0.1 to 0.5 , the number of users NRT is reduced by $22 \%$.

Now, we study the influence of $B R \_n r t$ on the number of accepted new real time calls. In Figure 8, we observe the reduction in the number of accepted new RT calls when $B R n c \_r t$ increases. However, this decrease is limited (about $9.8 \%$ ) because the blocking probability for new calls is only applied when the $t h \_R T \_N C$ is exceeded (above $75 \%$ of the bandwidth).

We notice thereafter that the number of RT HC accepted decreases as it depends on the number of preemptions. In fact, the more preemptions is performed, the higher number of RT $\mathrm{HC}$ accepted is obtained in the LTE system. However, as the RT HCs have the highest priority, we note that the decrease of the number of calls accepted of this type does not exceed $0.9 \%$.

\section{2) Investigation of BRnc_rt}

In this section, we investigate the influence of the blocking rate for RT NC $\left(B R n c_{-} r t\right)$. We notice on the one hand that the number of RT NCs slightly decreases when increasing BRnc_rt (see Figure 9).

On the other hand, the number of RT HCs increases as this type of calls has the highest priority and therefore it can profit from the increase of the blocking probability of RT NCs as well as from the preemption algorithm applied on NRT calls.

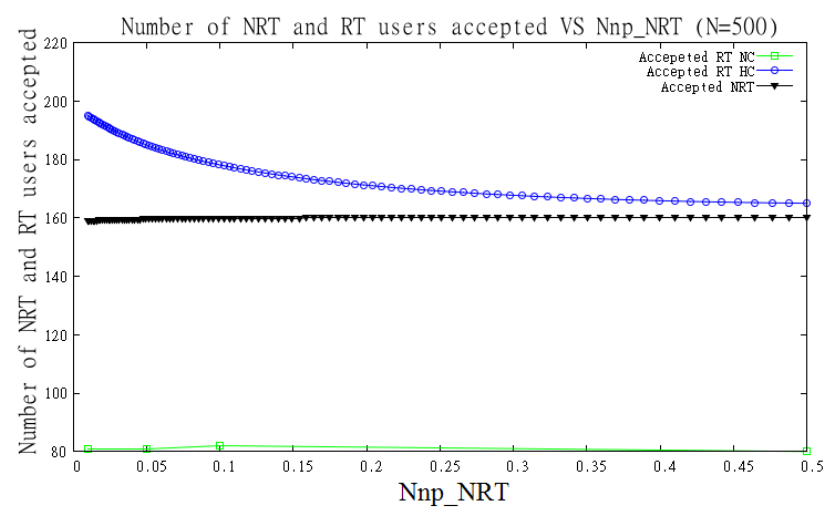

Fig. 10. Number of NRT and RT users accepted Vs Nnp_NRT

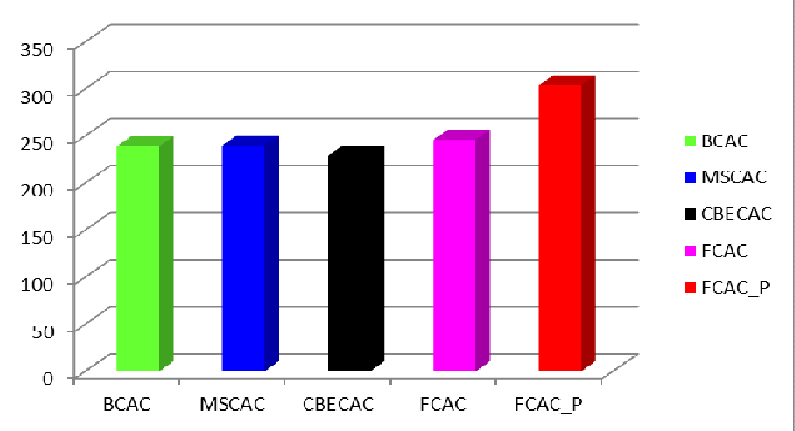

Fig. 11. Number of RT users accepted for different CAC mechanisms $(\mathrm{N}=500)$

3) Investigation of Nnp_NRT

Figure 10 represents the number of users accepted as a function of Nnp_NRT. Recall that the number of RT HC users accepted depends on the number of preemptions. In addition, the number of preemptions decreases when Nnp_NRT increases. Hence the number of RT HCs accepted decreases when increasing the number of non-preempted NRT users because the preemption algorithm is canceled when the number of non-preempted NRT users reaches Nnp_NRT.

We also verify that the number of NRT users accepted increases when Nnp_NRT increases as this parameter keeps NRT users in the LTE system

\section{CAC mechanisms comparaison}

We now compare the performance of different CAC mechanisms.

Figure 11 represents the average number of RT NC and RT HC users accepted when using FCAC_P, FCAC, BCAC, MSCAC and CBECAC.

Note that our proposed mechanisms provide the highest number of RT calls accepted because our CAC mechanism is flexible since it uses using different blocking rates. Moreover, it gives priority to RT users and reduces of the number of accepted users having bad channel. In fact, when the channel quality is bad, users cannot use efficient MCS and therefore require more PRBs (see Table I). We also verify that FCAC_P serves more RT users than FCAC; thanks to the preemption algorithm that expands the bandwidth allocated to users having higher priority. 
Figure 14 represents the number of NRT users accepted for the different CAC mechanisms. We note that FCAC_P and FCAC provide almost the same number of NRT users accepted compared to BCAC; thanks to the parameter th_NRT that provides resources to this type of calls in spite of the favoritism given to the RT calls by our FCAC mechanism. Finally, we notice that CBECAC serves more NRT users than MSCAC. In fact, on the one hand CBECAC uses Nnp_NRT to limit the preemption applied to NRT users. On the other hand, MSCAC applies preemption on users having bad channel and these users can be RT users.

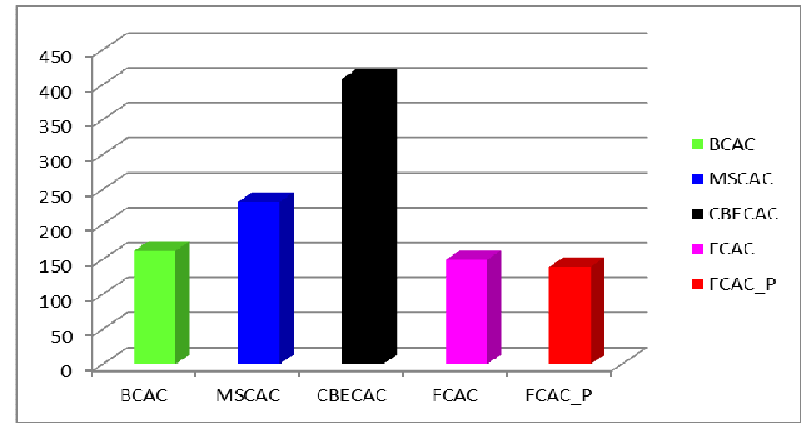

Fig. 14. Number of NRT users acceptes for different CACs

From the simultaion results decribed above, we can see that our flexible CAC scheme provides the highest number of accepted users having higher priorities. However, we have to check that our CAC mechanism deos not reduce the total throughput when favoring RT users.

Table IV presents the mean throughput for the different CAC mechanisms. We note that FCAC_P provides the highest system throughput because it takes into accont the quality of the channel in its CAC decision. Moreover, its flexibility characteristic using blocking probabilities allows the system to contain a large number of calls accepted. Note that the enhancement of the system throughput is more important when increasing the total number of users (1000 users instead of 500 users) as our flexible CAC can easly profit from the diversity of users.

Finally, we observe that BCAC and CBECAC provide low throughput when the number of users is equal to 1000 as these CAC mechanisms do not consider the quality of the channel in their CAC decisions.

TABLE IV. MEAN THROUGHPUT OF THE SYSTEM

\begin{tabular}{|l|l|l|}
\hline CAC mechanism & 500 users & 1000 users \\
\hline BCAC & $6.68 \mathrm{Mbit} / \mathrm{s}$ & $18.03 \mathrm{Mbit} / \mathrm{s}$ \\
\hline MSCAC & $6.86 \mathrm{Mbit} / \mathrm{s}$ & $24.25 \mathrm{Mbit} / \mathrm{s}$ \\
\hline CBECAC & $6.93 \mathrm{Mbit} / \mathrm{s}$ & $23.05 \mathrm{Mbit} / \mathrm{s}$ \\
\hline FCAC & $6.58 \mathrm{Mbit} / \mathrm{s}$ & $24.33 \mathrm{Mbit} / \mathrm{s}$ \\
\hline FCAC_P & $7.38 \mathrm{Mbit} / \mathrm{s}$ & $25.96 \mathrm{Mbit} / \mathrm{s}$ \\
\hline
\end{tabular}

\section{CONCLUSION}

Service providers should ensure total connectivity of their mobile users especially when a user moves from one network to another while providing the required amount of bandwidth and avoiding the termination of service.

In this paper, we devised new CAC schemes and evaluated their performance for RT and NRT classes of service of LTE system. We showed that FCAC_P benefits from the diversity of services. Moreover, this flexible CAC mechanism takes into account the congestion periods and does not totally reject user calls before profiting from the whole bandwidth. Moreover, our CAC mechanisms provide the highest system throughput as they take into account the quality of channel when deciding to accept a call and use dynamic thresholds of bandwidth.

In future work, it is interesting to specify the behavior of the CAC when a handoff call comes from another type of networks such as Wireless Local Area Networks (WLANs) [13]. Moreover, we aim to enhance the preemption algorithm taking into account additional parameters as the channel quality of preempted users as well as the number of PRBs already allocated.

\section{References}

[1] 3GPP Technical Specification, "E-UTRA, Radio Resource Control (RRC), Protocol specification”, TS 36.331 version 12.5.0 Release 12, April 2015

[2] 3GPP, "Overview of 3GPP Release 10 V0.2.1", June 2014

[3] 3GPP, "Overview of 3GPP Release 11 V0.2.0", 3GPP, September 2014

[4] Ericsson, "On the pulse of the networked society", Ericsson mobility report, June 2014

[5] M. Khabazian, O. Kubbar, and H. Hassanein, "Call admission control with resource reservation for multi-service OFDM networks", International Conference on Computing, Networking and Communications (ICNC), p. 781 - 785, Maui, HI, 30 Junuary - 2 Ferbruary 2012

[6] Jie Wang and Yangfan Qiu, "A New Call Admission Control Strategy for LTE Femtocell Networks", International Conference on Advances in Computer Science and Engineering (CSE 2013), Sydney, Australia, December 2013

[7] J. Vijay Franklin1 and K. Paramasivam, "Efficient Channel State Based Call Admission Control for Non Real Time traffic in LTE (3GPP) Networks", International Journal of Computer Science Issues, Vol. 9, Issue 2, No 2, March 2012

[8] A. Triki and L. Nuaymi, "InterCell Interference Coordination Algorithms in OFDMA wireless systems", IEEE VTC-Spring, Budapest, Hungary, May 2011

[9] Aymen Belghith, Selem Trabelsi, and Bernard Cousin, "Realistic Percategory Pricing Schemes for LTE users", 12 ${ }^{\text {th }}$ Intl. Symposium on Modeling and Optimization in Mobile, Ad Hoc, and Wireless Networks, WiOpt 2014, Hammamet, Tunisia, 12 - 16 May 2014

[10] 3GPP Technical Specification, "EUTRA and EUTRAN overall description, Stage 2", TS 36.300 V12.5.0, Release 12, April 2015

[11] Aymen Belghith, Wafa Ben ElMabrouk, and Selem Trabelsi, "Performance Evaluation of Mixed Traffic Scheduling Algorithm in LTE", the $14^{\text {th }}$ IEEE International Symposium on Network Computing and Applications, NCA 2015, Cambridge, MA, USA, 28 - 30 September 2015.

[12] Salem Trabelsi, Aymen Belghith, Faouzi Zarai, and Mohammad S. Obaidat, "Performance Evaluation of a Decoupled-level with QoS Aware Downlink Scheduling Algorithm for LTE networks", the $8^{\text {th }}$ IEEE International Conference on Internet of Things, iThings 2015, Sydney, Australia, 11 - 13 December 2015.

[13] Salwa Othmen, Faouzi Zarai, Mohammad S. Obaidat, and Aymen Belghith, "Re-authentication Protocol from WLAN to LTE", IEEE Global Communication Conference, GLOBECOM 2013, Atlanta, GA, USA, 9 - 13 December 2015. 